\title{
La Suisse du Nord-Ouest : un espace frontalier dans le processus de la mondialisation
}

The North-Western Switzerland in the process of globalization

Nordwestschweiz : ein Grenzraum im Prozess der Globalisierung

\section{Renato Strassmann}

\section{CpenEdition}

Journals

Édition électronique

URL : http://journals.openedition.org/rge/2804

DOI : $10.4000 /$ rge.2804

ISSN : 2108-6478

Éditeur

Association des géographes de l'Est

Édition imprimée

Date de publication : 1 janvier 2002

ISSN : 0035-3213

\section{Référence électronique}

Renato Strassmann, « La Suisse du Nord-Ouest : un espace frontalier dans le processus de la mondialisation », Revue Géographique de l'Est [En ligne], vol. 42 / 1-2 | 2002, mis en ligne le 05 janvier 2011, consulté le 07 septembre 2020. URL : http://journals.openedition.org/rge/2804 ; DOI : https:// doi.org/10.4000/rge.2804

Ce document a été généré automatiquement le 7 septembre 2020

Tous droits réservés 


\section{La Suisse du Nord-Ouest : un espace frontalier dans le processus de la mondialisation}

The North-Western Switzerland in the process of globalization

Nordwestschweiz : ein Grenzraum im Prozess der Globalisierung

\section{Renato Strassmann}

\section{Introduction}

1 La mondialisation entraîne une restructuration économique qui n'est certes pas propre à l'espace étudié, mais concerne l'ensemble de la Suisse. La Suisse du Nord-Ouest étant toutefois le principal pôle industriel helvétique (Füeg, 2000), elle ressent tout particulièrement cette restructuration (Strassmann, 2001). Celle-ci se traduit entre autres par des licenciements massifs dans les grandes entreprises, le transfert d'emplois des pays à salaires élevés vers les pays à salaires faibles, l'augmentation du taux de chômage, la réorganisation des entreprises et des exigences changeantes en qualification professionnelle (Strassmann \& Standke, 1998). Un important groupe de firmes à vocation est concerné par ces mutations : des producteurs industriels tels que Novartis, Hoffmann-La-Roche, Synthes-Stratec, Syngenta, Clariant, Sulzer Medica, Lonza Group, ABB (Asea-Brown-Bovery); mais aussi des groupes bancaires UBS (Union de banques suisses) et Crédit Suisse Group. La cause de la mutation structurelle réside dans l'accentuation de la mobilité des facteurs de production flexibles (Probst, 1997), influencée par :

- les technologies de l'information et de la télécommunication. Les progrès dans ce domaine augmentent la transparence du marché et incitent à exploiter de manière renforcée les avantages offerts par les coûts comparatifs dans le cadre du « global sourcing ";

- le développement du système de transport. L'aménagement des infrastructures, parallèlement à la réduction des coûts de transport et aux progrès réalisés dans le 
domaine de la logistique et des technologies du transport, conduisent à la réduction des résistances spatiales ;

- la suppression des barrières douanières et commerciales. Dans le cadre du GATT/OMC, les tarifs douaniers diminuent dans un nombre croissant de pays et pour de plus nombreux groupes de produits;

- I'intégration régionale. La formation de blocs économiques régionaux (UE, ALENA, ASEAN) induit une mobilité accentuée des personnes, des biens, du capital et des services ;

- des taux de change flexibles. L'abandon du système des taux de change fixes entraîne une mobilité renforcée du capital. La valeur élevée du franc suisse « pénalise » en outre la devise nationale en matière d'échanges internationaux, ce qui a incité un certain nombre de firmes à s'orienter vers les marchés des régions du globe à bas salaires ;

- de nouveaux marchés et de nouvelles offres. L'affirmation des Nouveaux pays industrialisés favorise entre autres l'apparition de nouveaux débouchés dans le Sud-est asiatique.

- la pénurie et le coût élevé du foncier. A l'étroitsur leur territoire, les entreprises du périmètre étudié sont conduites à contrer la pénurie en terrains, associée à une augmentation du prix du foncier préjudiciable à la compétitivité internationale. La pression résidentielle et entrepreneuriale sur le foncier suisse, et sur celui du Nord-Ouest en particulier, pénalise jusqu'aux terres agricoles les plus fertiles, à tel point que le signal d'alarme est devenu général (Office fédéral de la statistique, 2001). Les firmes procèdent ainsi à des implantations à l'extérieur des frontières nationales, soit dans la « Regio TriRhena » même, soit ailleurs en Europe, soit, surtout, dans les Nouveaux pays industrialisés.

\section{Mondialisation et transformation des structures spatiales. Le cas de la Suisse du Nord-Ouest}

2 La réduction des coûts de transport et le renforcement corrélatif de la concurrence mondiale, comme le fait de privilégier le savoir-faire aux dépens des matières premières, des sources d'énergie et du travail, font régresser en importance les facteurs traditionnels de production. Les travailleurs qui ont des connaissances générales et spécialisées dans des domaines adéquats conformes aux nouvelles orientations sont particulièrement recherchés (Porter, 1999). Le savoir-faire, en tant que facteur de production, joue aussi un rôle croissant en Suisse du Nord-Ouest par suite de l'accentuation de la division internationale du travail et du progrès technologique (fig. 1). Deux types de changements sont ainsi intervenus: des changements structurels économiques et une réorganisation spatiale. 
Figure 1 : Restructuration régionale de la Suisse du Nord-Ouest dans le cadre de la mondialisation

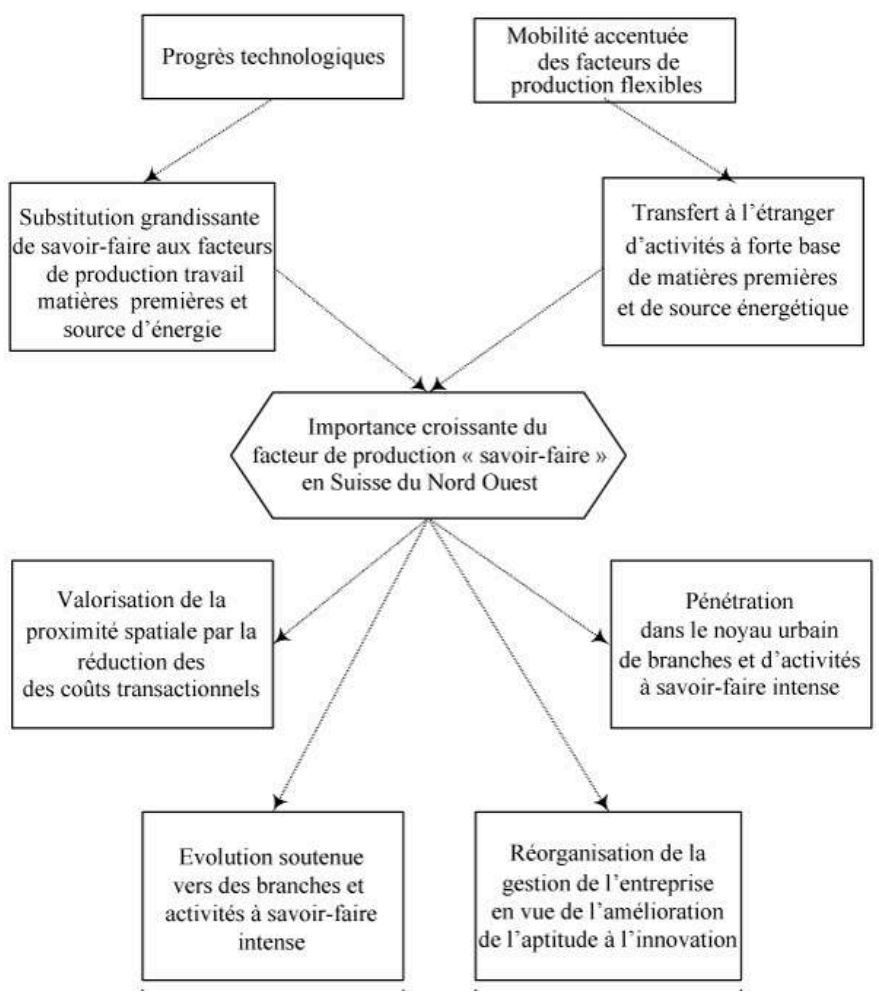

3 En ce qui concerne les changements structurels économiques, les mesures prises en matière de réorganisation entrepreneuriale (transformation du système hiérarchique traditionnel par la mise en place de hiérarchies "horizontales", décentralisation des entreprises, focalisation sur des compétences fondamentales, création de réseaux entrepreneuriaux), visent à la suppression des obstacles à la circulation de l'information et de l'optimisation de la circulation de cette ressource-clé. Ce procédé est destiné à améliorer l'aptitude innovatrice de l'entreprise et à garantir la survie de celle-ci dans un environnement hautement dynamique. A l'échelle de l'économie régionale, il a comme répercussions la réduction des effectifs de maind'œuvre, une tertiairisation croissante et la promotion des petites et moyennes entreprises.

4 L'importance accrue du savoir-faire en tant que facteur de production implique une réorganisation spatiale fondée sur le transfert des activités ayant surtout recours à la main-d'œuvre, à l'énergie et aux matières premières en direction des pays à faible salaire ou de la périphérie urbaine, tandis que des activités à savoir-faire intense s'installent en centre-ville. L'objectif de la présente contribution consiste à montrer de quelle manière ces tendances théoriques générales s'appliquent aussi à la Suisse du Nord-Ouest, principale région économique de la Confédération.

L'internationalisation économique entraîne un processus de valorisation de la région à hauts salaires que constitue la Suisse du Nord-Ouest, processus dans lequel le facteur de production "savoir-faire " occupe une position centrale. La mutation se manifeste par une tendance à l'émergence de branches et d'activités à savoir-faire intense, à la réorganisation des entreprises vers l'innovation, à la pénétration de branches et d'activités à important savoir-faire en centre-ville, de même qu'à la valorisation de la 
proximité spatiale au point de vue de la minimisation des coûts de transaction (Strassmann, 2001).

6 Le périmètre de cette étude comprend les demi-cantons de Bâle-Ville et de BâleCampagne, des portions des cantons de Solothurn (circonscriptions de Thierstein et Dorneck), les circonscriptions Rheinfelden et Laufenburg du canton d'Aargau, ainsi que les communes d'Elfingen, Bözen et Effingen qui appartiennent à la circonscription de Brugg (fig. 2).

Figure 2 : L'espace économique de la Suisse du Nord-Ouest

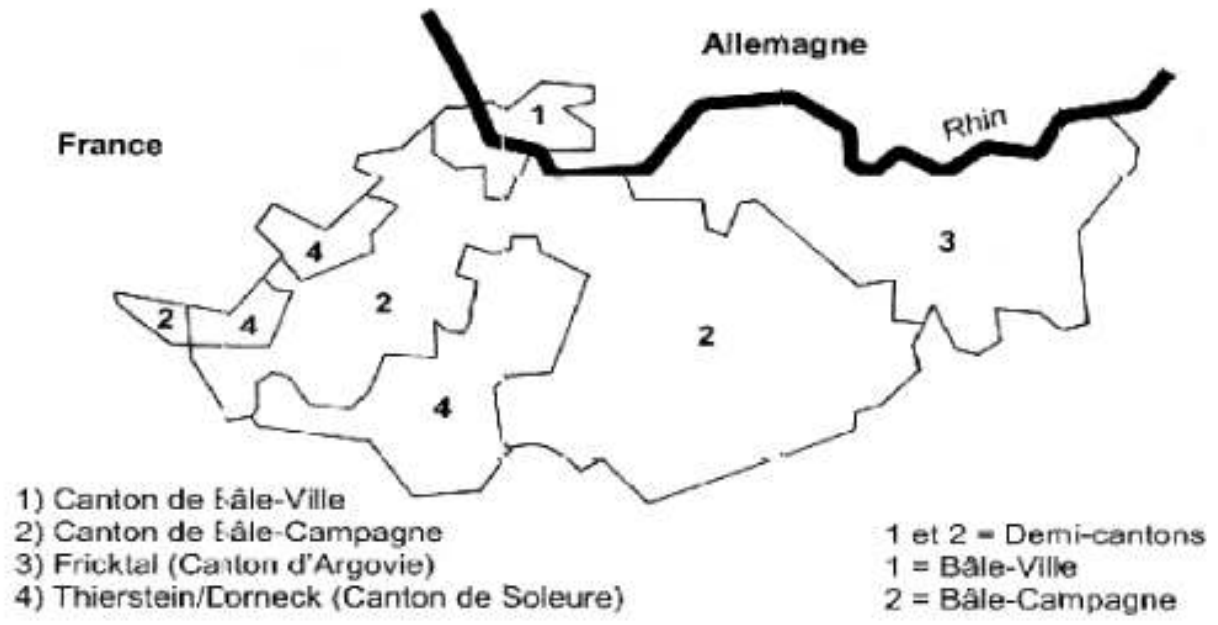

Source : Fueg R., 2001

7 La tranche chronologique retenue porte sur la période qui va de 1991 à 1998, parce qu'après 1989 , le processus de transformation économique a été fortement accéléré. L'étude porte uniquement sur les secteurs secondaire et tertiaire. Le secteur primaire n'ayant pas donné lieu à une enquête complète lors du recensement des entreprises, il n'a pu être pris en compte. La base de données repose fondamentalement sur le recensement confédéral des entreprises des années 1991, 1995 et 1998. Les transferts intra-régionaux en Suisse du Nord-Ouest sont observés dans le cadre des trois ensembles spatiaux constitués par le noyau urbain, l'agglomération et la périphérie, dont les principales caractéristiques sont rappelées dans le tableau 1.

Tableau 1 : Caractéristiques de la Suisse du Nord-Ouest et de ses aires régionales

\begin{tabular}{|l|c|c|c|c|}
\hline & Noyau urbain & Agglomération & Périphérie & Total \\
\hline Nombre d'habitants (1998) & 173216 & 270195 & 97527 & 540938 \\
Superficie en ha & 2385 & 33111 & 65584 & 101080 \\
Population/ha (1998) & 72,6 & 8,2 & 1,5 & 5,4 \\
Nombre d'emplois (1998) & 144470 & 116936 & 32843 & 294244 \\
Nombre d'emplois/habitant (1998) & 0,83 & 0,43 & 0,34 & 0,54 \\
Part d'emplois dans les grandes firmes & $31,7 \%$ & $13,3 \%$ & $13,7 \%$ & $22,4 \%$ \\
(>250 emplois à plein temps) & $74,6 \%$ & $64,3 \%$ & $46,5 \%$ & $67,4 \%$ \\
\hline Part du secteur tertiaire (1998) & & & & \\
\hline
\end{tabular}

Sources : Office Fédéral de Statistique, Statistik des jährlichen Bevölkerungsstandes 1998 ;

Bundesamt für Statistik, Eidgenössische Volkszählung 1990 ; Bundesamt für Statistik, Arealstatistik Schweiz ; Bundesamt für statistik, Eidgenössische Betriebszählung 1998 ; calculs personnels. 


\section{Transformation des structures économiques}

\section{La réduction du nombre d'emplois}

8 En Suisse du Nord-Ouest, le nombre d'emplois a diminué de 29894 unités, soit 9,2\%, entre 1991 et 1998 (tableau 2). Les causes en sont :

9 - le déploiement des technologies de l'information et de la communication. Les progrès technologiques tels que la mise en place des caissesscanner, le paiement électronique, conduisent à l'élimination de la main-d'œuvre dans les activités dites routinières (fonctions appelées « back office ») ;

10 - le renforcement du recours aux avantages de la grande taille. Des produits à forte intégration de savoir-faire, tels que ceux de l'industrie pharmaceutique, du software ou des nouveaux services bancaires, nécessitent des investissements énormes, poussant à augmenter la clientèle, à procéder à des fusions d'entreprises, dont les effets de synergie conduisent souvent à la réduction de la maind'œuvre ;

11 - le transfert d'activités à faible savoir-faire dans les pays à bas salaires;

12 - la diminution de l'offre en main-d'œuvre, qui est la conséquence de la prolongation du temps de formation initiale, de l'amplification de la formation permanente, des retraites anticipées ;

13 - le poids des produits courants. Les pertes d'emploi des branches de production courante ne sont compensées par des activités d'avenir que dans une proportion réduite; le renouvellement des effectifs se réduit ou se tarit. De plus, la recherchedéveloppement s'applique surtout aux marchés internationaux en stagnation (Borner, Brunetti \& Straghaar, 1990), ou se déploie de manière renforcée à l'étranger (Hotz-Hart, 1998);

14 - l'accentuation de la valorisation des investissements financiers. Par suite de la dérégulation et de l'internationalisation des marchés financiers, les investissements augmentent dans les affaires purement financières au détriment de ceux de la production réelle (Kneschaurek, 1999). Cette évolution ralentit la modernisation de l'appareil de production et la création d'emplois nouveaux ;

15 - le marché du travail (fig. 3). Dès leur origine, c'est-à-dire à la fin des années 1950, les migrations transfrontalières ont contribué à résoudre commodément - le taux de change attrayant et croissant de la devise helvétique aidant - une crise de sous-emploi dans les régions française et allemande voisines tandis que la main-d'œuvre manquait en Suisse du Nord-Ouest. A présent, ce qui a été considéré initialement comme un palliatif est devenu un élément structurant de la Regio TriRhena. La Suisse du NordOuest s'est habituée à la pénurie en effectifs nationaux et a délocalisé ses entreprises au-delà de ses frontières. N'empêche que son économie, notamment celle des deux Cantons de Bâle, ne saurait se déployer sans l'apport des migrations quotidiennes transfrontalières de travail. La restructuration en cours, qui s'appuie sur la mondialisation pour limiter autant que possible le nombre d'emplois par le passage à une économie de technologies avancées, est néanmoins en train de freiner ce mouvement. Les flux quotidiens en direction de la Suisse du Nord-Ouest concernent actuellement 31400 Français et 21000 Allemands. Les flux intra-suisses en direction de cette même région montrent combien le territoire national demeure pauvre en apports 
d'effectifs : 2000 flux émanent du Canton et de la République du Jura, 1000 de l'espace économique du Mittelland. Par contre 4000 ressortissants de la Suisse du Nord-Ouest vont travailler journellement dans l'espace économique de Zurich.

Tableau 2 : Evolution du nombre d'emplois en Suisse du Nord-Ouest par type d'espace (1991-1998)

\begin{tabular}{|l|c|c|c|c|c|}
\hline \multirow{2}{*}{ Type d'espace } & \multicolumn{5}{|c|}{ Evolution du nombre d'emplois } \\
\cline { 2 - 6 } & \multicolumn{2}{|c|}{1991} & \multicolumn{2}{c|}{1998} & Solde \\
\cline { 2 - 6 } & Chiffres absolus & Ch. Relatifs & Ch. absolus & Ch. Relatifs & Ch. abs. \\
\hline Noyau urbain & 168397 & $52,0 \%$ & 144470 & $49,1 \%$ & -23927 \\
Agglomération & 121151 & $37,4 \%$ & 116931 & $39,7 \%$ & -4220 \\
Périphérie & 34590 & $10,6 \%$ & 32843 & $11,2 \%$ & -1747 \\
Total & 324138 & $100.0 \%$ & 294244 & $100 \%$ & -29894 \\
\hline
\end{tabular}

Sources : Office Fédéral de Statistique, Recensement des entreprises 1995 et 1998 ; recherches propres de l'auteur

Figure 3 : Les flux de travail transfrontaliers en Suisse du Nord-Ouest (1999)

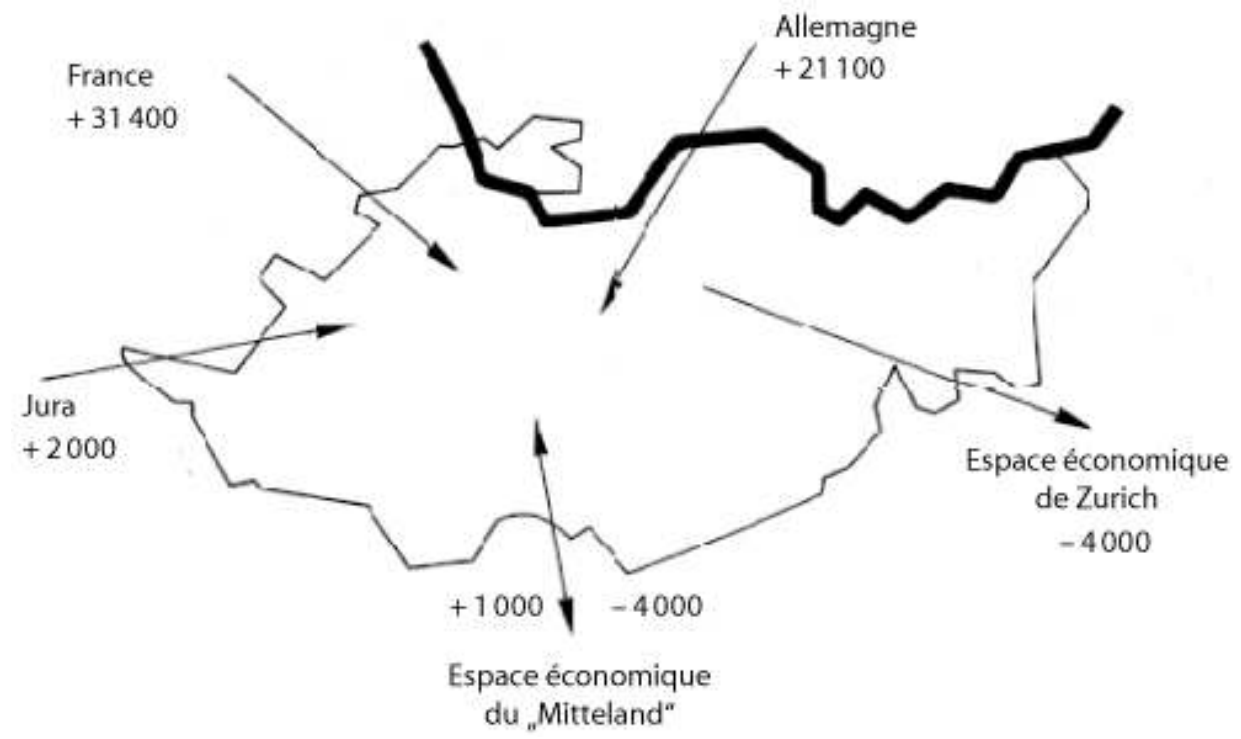

Source : Fueg R., 2001

\section{La croissance du secteur tertiaire}

Avec une régression de 22,9\% (- 28697 emplois), le secteur secondaire -bâtiment et travaux publics inclus- a perdu davantage en importance que le secteur tertiaire, dont le recul est relativement insignifiant : 0,7 \%, soit - 1431 emplois. La mutation en cours est favorable au secteur tertiaire, dont le taux d'emplois par rapport au nombre total est passé entre 1991 et 1998 de 61,6\% à 67,4\%. L'importance accordée au secteur tertiaire s'explique entre autres par les évolutions suivantes :

- I'« outsourcing» de soi-disant personnes juridiques. Il se traduit par le transfert, en provenance des firmes multinationales, de fonctions considérées comme banales vers des PME indépendantes, en vue de réduire les coûts et les risques entrepreneuriaux. Comme de nombreux domaines externalisés (nettoyage, informatique, calcul, logistique...) ont appartenu précédemment au secteur secondaire, 
et sont à présent partie prenante du secteur tertiaire, celui-ci augmente en importance sans que l'activité elle-même n'ait subi de changement. Ce transfert n'est pas uniquement interne à la Suisse, mais concerne aussi le voisinage français et allemand, ainsi que l'étranger lointain, où il se mêle aux délocalisations, suscitant des enchevêtrements complexes et multiples, dont l'étude reste à faire ;

- la modification du comportement en matière de demande. L'augmentation des effectifs dans le domaine de la santé, des affaires sociales, de l'informatique, de l'enseignement, des télécommunications et de l'immobilier est la conséquence du transfert de la demande vers le secteur des services. Cette évolution explique, selon Jean Fourastié, le déplacement croissant de la demande, dans un premier temps, du secteur primaire vers le secteur secondaire, puis de celui-ci vers le secteur tertiaire.

Dans ce contexte, la Suisse du Nord-Ouest est tout particulièrement caractérisée par l' augmentation de l'importance des branches et des activités à haut niveau de savoir-faire, celui-ci pouvant être défini, dans notre analyse, comme étant à base d'études supérieures de troisième cycle effectuées soit en formation fondamentale, soit partiellement ou entièrement en formation continue.

Entre 1991 et 1998, les gains les plus importants en matière d'emplois sont ceux (tableau 3) des branches sanitaire et sociale (3 523), de conseil en entreprise (2 728), d'auxiliaires de transport (2 481), de services informatiques (2 291), d'enseignement (1 274) et de services financiers divers (935).

Tableau 3 : Evolution de l'emploi dans les branches « gagnantes » de la Suisse du Nord-Ouest

\begin{tabular}{|l|r|r|r|}
\hline & \multicolumn{2}{|c|}{ Nombre d'emplois } & \multirow{2}{*}{ Solde } \\
\cline { 2 - 3 } & \multicolumn{1}{|c|}{1991} & 1999 & \\
\hline Santé et domaine social & 28898 & 32421 & 3523 \\
Conseil en entreprise & 21985 & 24713 & 2728 \\
Auxiliaires de transport & 5456 & 7937 & 2481 \\
Enseignement & 13724 & 14998 & 1274 \\
Services informatiques & 1945 & 4236 & 2291 \\
Télécommunications & 6033 & 6552 & 519 \\
Services financiers & 344 & 1279 & 935 \\
\hline
\end{tabular}

Sources : Office Fédéral de Statistique, Recensements des firmes 1991 et 1998 ; enquêtes de l'auteur

Ces branches ont ceci en commun que le traitement de l'information et du savoir-faire occupe une position centrale, qu'elles produisent des biens immatériels (santé, information, logistique et formation), qu'il s'agit de branches à croissance globale (santé et action sociale, conseil en entreprise, enseignement, services informatiques, télécommunications), que les services sont hautement 
spécialisés et souvent personnels (santé et action sociale). Par là-même, elles ne peuvent être remplacées que de façon limitée par les technologies de l'information et de la communication.

A l'intérieur des branches elles-mêmes, nous observons des glissements vers des activités à savoir-faire élevé (Neue Zürcher Zeitung, 19/02/2002). Cette évolution est tout particulièrement expressive dans l'industrie chimique (tableau 4). Les fabrications à faible création de valeur sont de plus en plus localisées sur des aires favorables à des coûts salariaux et environnementaux réduits. Les pertes d'emplois dans le domaine des éléments pharmaceutiques de base (- 6 828) et celui d'autres fabrications chimiques (6591 ) constituent des indicateurs de cette évolution. La Suisse du Nord-ouest est focalisée sur des orientations à création intensive de valeur, telle que celle des médicaments (9678), l'industrie chimique se transformant toujours davantage en industrie pharmaceutique. Le terme "chimie", encore courant, y compris dans les statistiques officielles globales, prête ainsi à confusion. Le tableau 4 donne autant de précisions que possible sur la nouvelle ventilation actuelle de cette industrie dite chimique. Nous apercevons par contre que la progression des emplois apparait principalement dans les biotechnologies, à base de fabrication de médicaments et de produits agro-chimiques, voire de produits de peinture; les produits de lessive, de nettoyage, les déodorants et soins du corps sont secondaires. Il est donc possible de déceler dans les statistiques officielles, pour l'essentiel sinon encore de manière très fine, la part des biotechnologies dans la Regio TriRhena et au-delà.

Tableau 4 : Evolution des emplois dans l'industrie chimique en Suisse du Nord-Ouest

\begin{tabular}{|l|c|r|r|}
\hline \multirow{2}{*}{\multicolumn{1}{|c|}{ Activité }} & \multicolumn{2}{|c|}{ Nombre d'emplois } & \multirow{2}{*}{ Solde } \\
\cline { 2 - 4 } & 1995 & 1998 & -5389 \\
\hline Produits de base chimiques & 8209 & 2820 & 729 \\
Produits agro-chimiques & 1114 & 1843 & 2608 \\
Produits de peinture et mastic & 711 & 3319 & -6828 \\
Produits de base pharmaceutiques & 9423 & 2595 & 9678 \\
Médicaments & 5286 & 14964 & 7 \\
Produits de lessive, de nettoyage : & 788 & 795 & -6625 \\
déodorants et soins du corps & 7095 & 470 & -5820 \\
Autres produits chimiques & 36626 & 26806 & \\
\hline Total & & & \\
\hline
\end{tabular}

Sources : Office Fédéral de la Statistique, recensements des entreprises de 1995 et 1998 ; calculs de l'auteur

Selon la Banque nationale suisse, les investissements suisses à l'étranger ont augmenté de $20 \%$ environ en 2001, ce qui correspond à un montant de 372,6 milliards de Francs suisses. Cet accroissement est surtout inhérent aux groupes dits « chimiques », dont les sièges, essentiellement localisés à Bâle, ont investi les trois-quarts de ce montant dans le secteur industriel. La mise en relation des investissements à l'étranger et des réductions d'effectifs en Suisse depuis 1997 confirme la politique de délocalisation. La fusion des firmes Ciba et Sandoz, passées de la production chimique à la production pharmaceutique, en un groupe appelé Novartis (novembre 1995), a conduit à l'annonce de la suppression de 10000 emplois dans l'ensemble de la Suisse et à celle d'un vaste programme d'investissements à l'étranger. A partir de 1996, la suppression d'emplois en Suisse du Nord-Ouest s'est poursuivie à Pratteln, dans la firme Schindler 
(construction de wagons) et dans la filiale de Daimler-Benz, Adtranz, à Muttenz, pour un total de 3000 emplois...

Une étude publiée en 2001 (Füeg, 2001) montre que le coût du travail/emploi s'est réduit dans l'industrie de la Suisse du Nord-Ouest de près de $5 \%$ par suite de la compression des effectifs, alors qu'il a augmenté dans les PME de $2,3 \%$, voire, dans certaines branches, de $14 \%$, car ce mouvement s'accompagne d'une augmentation de l'importance des petites et moyennes entreprises. En Suisse du NordOuest, la part prise par les microentreprises (0-9 emplois à plein temps) apparaît nettement: tandis que dans le secteur secondaire n'ont été créées que $26(+0,2 \%)$ possibilités d'emploi, le secteur tertiaire en a enregistré 3 438. Les causes de cette évolution s'expliquent par :

- l'importance croissante des réseaux. Les cycles de production raccourcis, la mutation économique, écologique et sociale plus rapide, ainsi que la tendance à la fourniture sur mesure des biens et des services, exigent une plus grande aptitude d'adaptation et de réaction des entreprises, plus facile à obtenir dans des délais brefs en PME. La Suisse du Nord-Ouest met à profit son appartenance à un espace trinational pour étendre ses réseaux au-delà des frontières nationales et bénéficier ainsi d'avantages productifs dans le reste du Rhin Supérieur méridional, considéré à présent comme une "Biovalley " : si selon la "Basler Zeitung " (24.5.2000), seule une faible part de ces capitaux ont bénéficié à la Biovalley, les initiatives prises en faveur des implantations d'entreprises dans la Biovalley ont été le fait des milieux économiques et politiques régionaux qui bénéficient d'aides de la part du programme «Interreg » de l'Union européenne. En la quasi-absence des trois multinationales jouant pleinement la mondialisation ("global players») - Aventis, Novartis, Roche - elles ont permis la création, depuis 1997, de 81 entreprises, dont 31 durant l'année de la fusion entre Ciba et Sandoz (Basler Zeitung du 24.5.2000);

26 - la perte de l'importance des avantages de grande taille. Les services satisfont de plus en plus directement la clientèle finale et tiennent compte des besoins de celle-ci. Par là-même, les avantages de taille inhérents aux économies d'échelle déclinent, la production sur mesure prenant le dessus; celle-ci peut être désormais aussi bien assurée par les PME que par les grandes ;

27 - les progrès réalisés par les technologies de l'information et de la communication permettent à présent de relier les PME entre elles en réseaux virtuels efficaces, de produire et d'offrir au marché globalisé des marchandises et des services à prix avantageux ;

28 - des marchés nouveaux. L'individualisation des besoins des clients, l'élargissement des marchés dans le cadre du processus de la mondialisation, l'« outsourcing » des grandes entreprises, la demande croissante de fret et de services par les grandes firmes créent des marchés nouveaux pour les PME.

29 En résumé, l'augmentation de l'importance du facteur de production "savoir-faire » pour l'efficacité des entreprises, les recentrages des compétences fondamentales et l'«outsourcing", obligent les firmes à mettre l'accent sur l'accélération et l'élargissement des flux de communication et de savoir-faire (hiérarchies horizontales, mise en réseaux intra- et extra-entreprises), l'optimisation du management informationnel (recours aux banques de données, management du savoir), ainsi que sur 
le degré de précision, le volume et le degré d'actualité des informations disponibles, en vue d'une amélioration de l'aptitude à l'innovation.

A l'échelle de l'économie régionale, cette évolution se traduit par la diminution de la taille moyenne des entreprises, l'augmentation du nombre de lieux de travail, la progression du secteur tertiaire, l'orientation vers des branches de production de haut niveau. Par là-même, l'économie régionale de la Suisse du Nord-Ouest adopte de façon croissante une structure de PME en réseaux.

\section{La réorganisation spatiale}

La Suisse du Nord-Ouest est soumise à d'importantes modifications spatiales, sans qu'elle ne soit affectée de manière significative par des pertes d'emplois ou de population dans son ensemble. Si dans certains secteurs d'activité et quelques communes, le nombre d'emplois ou d'habitants a diminué, le solde global est demeuré relativement stable. Avec 546000 habitants au total en 2000, la baisse de la population est demeurée insignifiante. Le Canton de Bâle-Ville a certes perdu 2700 habitants en 2000/2001, mais l'agglomération et son espace périurbain ont enregistré une augmentation correspondante. Tandis que les pertes de population ont été massives à Bâle-Ville dans un passé plus éloigné, les migrants se sont installés essentiellement à Bâle-Campagne.

Par son solde d'emplois et de population, quasiment inchangé depuis 1999, la Suisse du Nord-Ouest continue à rester le territoire économique le plus important de la Confédération, dans la mesure où les pertes d'emplois industriels ont été compensés en valeur ajoutée par des apports internes dans le secteur tertiaire. La part des travailleurs frontaliers demeure élevée et stable : $28,8 \%$ dans l'industrie, $15,8 \%$ dans les services, $38 \%$ dans l'alimentation et les boissons. Elle s'est même accrue légèrement en 2000/2001, tandis que les emplois occupés par des Suisses ont diminué en conséquence. (Füeg, 2001). Elle sert de régulatrice du marché de l'emploi pour limiter le recours à des effectifs suisses trop coûteux. Le taux de chômage est également faible : 1,6 \% en 2000, $1,9 \%$ en 2002 , contre un taux national de $2,1 \%$. Le "système " en place reste stable, alors que le territoire est soumis à des mutations sensibles. Celles-ci sont masquées par les statistiques officielles, insuffisamment expressives des changements intervenus ou en cours. Il est donc impossible de cartographier l'évolution fine des types d'emploi ou celle des flux internes. L'étude des taux d'emplois ou d'habitants rapportés à l'hectare reste à effectuer. A ce titre, pour quantifier et spatialiser autant que possible les phénomènes évoqués ici nous renvoyons le lecteur à l'article et aux cartes de R. Schneider-sliwa, en ce volume.

\section{La suburbanisation de l'activité économique}

33 La Suisse du Nord-Ouest n'a pas échappé à la périurbanisation (Nommel et al., 1999/2000). Nous observons d'abord un transfert d'emplois inhérent à la suburbanisation de la population (Schneider-Sliwa, 1999). De 1991 à 1998, le noyau urbain est le seul type d'espace à avoir enregistré une évolution négative de la population (- 5283 habitants). Le transfert d'emplois vers l'espace environnant s'explique par les causes suivantes : 
34 - l'approvisionnement suit la demande. Le commerce de détail, la réparation d'automobiles, le secteur de la santé et de l'action sociale ainsi que l'immobilier s'adaptent au transfert de la population et augmentent leur offre de services tant dans les communes du reste de l'agglomération que dans celles de la périphérie. Ce phénomène est également observable dans le domaine de l'évacuation des eaux usées et des déchets, comme dans ceux de la distribution d'énergie ou du loisir, ainsi que de la culture : là aussi l'offre de services est valorisée dans l'espace suburbain ;

- une création d'entreprises renforcée dans l'espace suburbain. Comme la création d'entreprises, notamment tertiaires, est souvent liée à la recherche de la proximité résidentielle (Hanser \& Meier, 1992), le transfert d'une partie de la population à revenus élevés et à haut niveau de qualification favorise cette créativité dans l'espace suburbain. C'est ainsi que de 1991 à 1998, le nombre de lieux de travail ne s'est accru que de 1,7\% dans le noyau urbain, alors que l'augmentation du nombre de ces lieux dans l'ensemble de l'aire urbaine a atteint $8,2 \%$;

- I'augmentation de l'attractivité de l'espace périurbain. La suburbanisation de la population contribue à une valorisation constante de l'espace suburbanisé. L'extension du système des voies de communication améliore tout particulièrement l'accessibilité des communes suburbaines. Cette évolution réduit l'écart entre le noyau urbain et l'espace suburbain en matière d'accessibilité.

La suburbanisation a eu également comme conséquence la restructuration économique du noyau urbain de Bâle. Entre 1991 et 1998, celui-ci a perdu 31,3\% des potentialités en matière d'emploi dans le secteur secondaire, contre $18,5 \%$ dans le reste de l'agglomération et 10,9 \% à la périphérie. Quoique la désindustrialisation libère de l'espace, les aires devenues ainsi disponibles ne peuvent être affectées à d'autres usages qu'au moyen d'investissements imposants. De plus, la pénurie d'espace devient un enjeu croissant : absence d'espaces libres, localisation de l'essentiel des terrains à proximité de la City, appartenance d'une part importante de la propriété foncière aux institutions publiques, demande soutenue de terrains par l'industrie chimique, diminution de l'intensité de l'usage du sol, valorisation de la ville en tant qu'aire résidentielle et centre de services.

Le manque d'espace accélère l'élimination dans le noyau urbain des activités à faible taux de création de valeur (le bâtiment, le commerce et la réparation d'automobiles, le commerce de détail), au profit d'usages à forte valeur ajoutée (l'informatique, le conseil en entreprise). Cette évolution est particulièrement perceptible en des lieux soumis à une pression croissante en matière de développement, comme c'est le cas à proximité de la gare centrale des Chemins de fer fédéraux (Gerheuser \& Perret-Gentil, 1993). De plus, la densité élevée d'entreprises fortement polluantes est devenue indésirable par des citadins aux revenus croissants, qui accordent désormais une importance grandissante à la qualité environnementale de leur voisinage. Cette tendance est accentuée par la valorisation de la ville en tant que centre résidentiel et de services. La présence d'une densité élevée de firmes, ainsi que le manque d'espaces libres, conduisent à un affaiblissement de la qualité de localisation et au ternissement de l'image urbaine. Pareille situation incite surtout les entreprises de services à s'abstenir d'une implantation sur des territoires marqués par les nuisances. 


\section{La centralité soutenue de Bâle}

39 La suburbanisation de l'activité économique ne doit toutefois pas faire oublier que le noyau urbain de la métropole continue à avoir une grande importance économique. De nombreux indicateurs révèlent cette situation :

- la concentration des emplois dans le noyau urbain. En 1998 49,7\% des emplois sont concentrés sur $2,4 \%$ de la surface totale de la Suisse du Nord-Ouest. Le noyau urbain de Bâle constitue ainsi le centre de gravité de la région ;

41 - la concentration de branches à haute valeur ajoutée dans le noyau urbain. La répartition spatiale des branches d'activité révèle une structure de production à valeur ajoutée élevée au centre de l'aire métropolitaine, alors que des branches de niveau inférieur sont davantage perceptibles dans l'espace environnant. Ainsi les emplois tertiaires sont nombreux au centre même de Bâle, contrairement à la périphérie dans laquelle ils sont plus clairsemés. Les services de haut niveau (compagnies aériennes, assurances, crédit, télécommunications) montrent un fort gradient centre/périphérie. Ils côtoient au centre le secteur de la distribution : activités aériennes, navigation, auxiliaires de transport, transports terrestres. Il est vrai que la distinction entre « haut niveau » et «niveau inférieur» est plus ou moins arbitraire, dans la mesure où le savoir et le savoir-faire de pointe sur lesquels s'appuie le " haut niveau» ne peuvent être appréhendés qu'approximativement, étant de plus en constante évolution. L'une des caractéristiques principales réside dans le fait qu'ils requièrent la mise en œuvre de technologies encore rares ou rarement appliquées qui assurent aux firmes concernées d'exceptionnelles plus-values ;

42 - la pénétration et la concentration d'activités de niveau élevé dans le noyau urbain. Le contraste entre le centre et la périphérie est également observé à l'intérieur de certaines branches, autant dans l'industrie chimique que dans les services aux entreprises. Dans l'industrie chimique, c'est la production finale de la chaîne de création de valeur qui est localisée dans le noyau urbain : fabrication de produits agrochimiques, produits de peinture, mastic et médicaments. La production intermédiaire, en amont de la chaîne (gaz industriels, couleurs et pigments, éléments organiques, textiles artificiels, produits de lavage et de nettoyage, produits odorants et d'hygiène du corps), est localisée dans le reste de l'agglomération et à la périphérie, où l'on fabrique aussi des engrais et des composants d'azote, de même que des matières de base pour l'industrie pharmaceutique. Les services aux entreprises intègrent des activités de plus en plus abstraites et à haut savoir-faire au fur et à mesure que l'on se rapproche du noyau urbain. Ici se regroupent cabinets d'avocats, études notariales, services des patentes et de leurs applications, services d'analyse économique et fiduciaires, entreprises de sondage d'opinion et de marchés, conseil en entreprise, conseil en publicité, courtage en publicité, courtage en main d'œuvre et en emplois.

Les activités abstraites et à savoir-faire intense ont ainsi une importance et un développement supérieurs à la moyenne dans le noyau urbain. Contrairement à une opinion courante, la centralité conserve tout son poids. Ce phénomène s'appuie sur les facteurs suivants :

44 - la présence d'un bassin de main-d'oeuvre. Les firmes qui nécessitent des emplois à haute qualification ont besoin d'un important bassin de main-d'œuvre et, par là-même, d'une centralité urbaine de niveau élevé ; 

pour faire face aux problèmes soulevés dans le noyau urbain, compte tenu de l'importance prise par le savoir-faire. Dans cette optique, la politique économique est appelée à veiller à une valorisation continue de l'économie régionale. Pareille attitude implique la substitution d'une politique de localisation à base d'innovation à celle fondée sur des principes factoriels. Ce changement de paradigme signifie l'abandon d'une recherche de ressources centrales meilleur marché. La réorientation politique favorise la restructuration des transports urbains et, par conséquent, l'amélioration qualitative des noyaux urbains. Le développement de l'aptitude innovatrice peut être sous-tendu par la poursuite du déploiement de la formation fondamentale et continue, de la création d'entreprises, de l'attention portée à la bonne santé du potentiel entrepreneurial en place, de l'incitation à une coopération régionale et interrégionale plus active, ainsi que du renforcement des forces vives locales.

La mutation de l'industrie chimique en industrie pharmaceutique, la croissance du secteur de la santé et de l'action sociale, les efforts de promotion des biotechnologies et des technologies relevant du génie génétique constituent des indicateurs relatifs à l'importance prise par la branche sanitaire dans l'espace de localisation de la Suisse du Nord-Ouest. Les forces vives locales sont appelées à répondre à une demande croissance en matière de santé (Nefiodow, 1999). La promotion de technologies durables, des initiatives spectaculaires dans le domaine de la formation et l'exploitation des potentialités de la branche sanitaire sont susceptibles de faciliter cette démarche. De nombreuses autres évolutions innovatrices vont dans le même sens, souvent fondées sur la tradition humaniste de la région. 


\section{BIBLIOGRAPHIE}

Borner S., Brunetti A., StraubhaAR T. (1990). - Schweiz AG, vom Sonderfall zum Sanierungsfall ? Zurich.

FÜEG R. (sous la direction de) (2000). - Wirtschaftsstudie Nordwestschweiz, 99, Helbing \& Lichtenhahnverlag, Basel, 62 p.

FÜEG R. (sous la direction de) (2001). - Wirtschaftsstudie Nordwestschweiz, Schriften der Regio, 7.23, Regio Basiliensis, Basel.

GERHEUSER F., PERRET-GENTIL J. (1993). - Gewerbeverdrängung - Gewerbeentwicklung und Gewerbepolitik in den schweizerischen Grossstädten, Zurich.

HANSER C., MEIER H. (1992). - Kommerzielle Dienstleistungen. Ein Schlüsselelement für die Entwicklung von Mittelstädten ? Zurich.

HOTZ-HART B. (1998). - Wo liegt das Schweizer Innovationspotential ?, dans Scheidegger A., Hofer H. \& Scheuenestuhl G. (sous la direction de) : Innovation - Venture Capital Arbeitsplätze. Antworten zu den Kernfragen, Berne, Stuttgart, Vienne, pp. 47-54.

Kneschaurek F. (1999). - Weltwirtschaft im Umbruch, Probleme, Analysen, Perspektiven, Zurich.

Nefiodow L.A. (1999). - Der sechste Kondratieff, Wege zur Produktivität und Vollbeschäftigung im Zeitalter der information, Sankt Augustin.

NEUE ZÜRCHER ZEITUNG, 19/20.1.2002 : Globalisierung im Rekrodtmepo. Ergebnisse der Schweizer Direktinvestitionsstatistik 2000, p. 23.

Nommel J.U., SANDTNER M., WAFFENSCHMidT C. (1999/2000). - « Von der “A-Stadt” sur "Agglomeration" ? Suburbanisierungs- und Counterurbanisierungsprozesse in Basel-Stadt und Basel-Landschaft », Wirtschafsstudien in der Nordwestschweiz, Sissach, pp. 133-141. OfFice fédÉrAl de la stATISTIQUe (2001). - Bodennutzung im Wandel. Arealstatistik Schweiz, Neuchâtel.

PORTER M.E. (1999). - Nationale Wettbewerbsvorteile, erfolgreich konkurrieren auf dem Weltmarkt, Munich

PROBST R.P. (1997). - « Strukturwandel in der Region - Herausforderung für KMU's », Wirtschaftsstudien Nordwestschweiz, 1996/97, Sissach.

SCHNEIDER-SLIWA R. et al. (1999). - Bevölkerungsstruktur und Bevölkerungsdynamik beider Basel, Coll. «Stadt und Region », 1, Statistisches Amt des Kantons Basel-Stadt, 56 p.

STRASSMANN R. (2001). - « Restrukturierung der Regionalökonomie der Nordwestschweiz vor dem Hintergrund der Globalisierung. Analysen, Strategien und Visionen für Regionalpolitik und Regionalentwicklung ", Basler Beiträge zur Humangeographie, 47, Allschwihl.

STRASSMANN R., STANDKE O. (1998). - « Wirtschaftswandel in der Nordwestschweiz. Dynamik und wirtschaftsgeographische Aspekte », Regio Basiliensis (Struktur und Dynamik in der Agglomeration Basel), 39/2, Bâle. 


\section{RÉSUMÉS}

Le processus de mondialisation économique conduit en Suisse du Nord-0uest à un renforcement de l'importance du savoir-faire en tant que facteur de production. Cette signification accrue du savoir-faire se manifeste par la croissance des branches d'activité à forte mobilisation de matière grise, notamment dans le secteur tertiaire. Elle renforce aussi l'intérêt témoigné aux petites et moyennes entreprises. Cette mutation structurelle est liée à une réorganisation spatiale qui se traduit par la suburbanisation des firmes et une division du travail grandissante entre le noyau urbain et l'espace environnant. La ville centrale assume de plus en plus des fonctions liées aux activités abstraites, à un niveau de conception élevé et à un savoir-faire intense. Une politique économique innovante pourrait sous-tendre cette orientation, améliorer par là-même l'aptitude concurrentielle de l'économie régionale et contribuer à résoudre les problèmes démographiques posés au noyau urbain de Bâle en évolution.

The forces of economic globalization have been increasing the importance of knowlegde and innovation as factors of production in North Western Switzerland. This is evident in the growth of R\&D-intensive firms, tertiary activities and small and medium size firms. As a concomittant of structural changes in the economy, the spatial reorganization manifests itself in the suburbanization of firms and the progressive functional division between central city and suburban fringe. The central city is developing as a hub of knowledge-based activities. Development policy that supports these trends towards a knowledge based economy could increase the competitiveness of the entire region and also contribute to the solution of the problems that demographic developments impose upon the central city of Basle.

Der Prozess der wirtschaftlichen Globalisierung führt in der Nordwestschweiz zu einem Bedeutungsgewinn des Produktionsfaktors Wissens, der sich in einem Wachstum wissensintensiver Branchen, tertiärer Tätigkeiten sowie einen Trend $\mathrm{zu}$ Klein- und Mittelbetrieben manifestiert. Verbunden ist dieser strukturelle Wandel mit einer räumlichen Reorganisation, welche sich in einer Suburbanisierung von Unternehmen und einer fortschreitenden Arbeitsteilung zwischen Kern und Umland zeigt. Die Kernstadt fungiert zunehmend als Standort abstarkter, dispositiver und wissensintensiver Tätigkeiten. Eine innovationsorientierte Wirtschaftspolitik könnte diesen Trend unterstützen und damit gleichermassen die Konkurrenzfähigkeit der Regionalökonomie erhöhen und einen Beitrag zur Lösung der Probleme der sich demographisch verändernden Kernstadt leisten.

\section{INDEX}

Keywords : Economic Policy, Globalization, Innovation, Knowlegde Society, North Western Switzerland

Schlüsselwörter : Globalisierung, Innovation, Nordwestschweiz, Produktionsfaktor Wissen, Standortfaktoren, Standortpolitik

Mots-clés : facteurs de localisation, innovation, mondialisation, politique de localisation, savoirfaire facteur de production, Suisse du Nord-Ouest 


\section{AUTEUR}

\section{RENATO STRASSMANN}

Docteur en géographie de l'Université de Bâle - Helvetia Patria Versicherungen - Chef du département « Prozesse und Qualität » - St. Alban-Anlage $26 \mathrm{CH}-4002$ Basel 